\title{
Passerelles. Dialogues d'écrivains autour du silence
}

Le silence a cette vertu de rendre les écrivains extrêmement prolixes à son propos. Est-ce conscience aiguë de cela qui borde leurs textes, en amont comme en aval, de ce vide dans lequel ils craignent plus ou moins de se perdre?

M'intéressera ici leur observation des relations entre silence et voix : anges qui passent dans les conversations, silence supposé du monde, des choses. Si au verbe le silence s'érige en absolu antagoniste, les contours de la frontière qui les sépare sont flous, indistincts - ce sont les écrivains qui nous l'apprennent.

\section{Un ange passe}

La conversation est une des formes les plus méconnues du silence (Gary, $1981: 134$ ).

Ils sont nombreux à avoir voulu traquer les non-dits qui compliquent les relations humaines, les silences qui s'y mêlent.

Une typologie pourrait commencer par ce que masque un flot de paroles. Certaines œuvres sont l'illustration de cette logorrhée, comme, milliers de pages d'une écriture minuscule (les fameux " microgrammes "), celle de l'écrivain suisse Robert Walser. Dans ces textes, se donne à voir "l'équivalence entre le silence et une certaine utilisation ornementale de la parole ", une sorte de "bavardage prolongé pour cacher le manque de n'importe quel pas en avant dans le discours " (Calasso, $1995: 36$ ).

Même flot de paroles et - même silence lourd chez Marina Tsvetaieva :

Que voulez-vous que je fasse du silence humain ? Il m'oppresse, m'abat, me renverse, je le meuble avec un contenu qui peut aussi être parfaitement inadéquat. [...] Je perds mon

Marc Courtieu - docteur ès lettres, mathématicien ; membre de l'Institut d'Études des Langues et Littérature Européennes (ILLE) de l'Université de Mulhouse Haute-Alsace ; email: marc.courtieu@ gmail.com 
naturel, [...] je deviens complètement vide, tout entière concentrée sur une seule préoccupation : ne pas laisser l'air de la pièce se taire (2008: 351).

La poétesse russe l'écrit ; il s'agit, à chaque fois, de meubler, comme du regard de l'autre on détourne le sien. N'est-ce pas de ce jeu entre indifférence et hostilité, dissimulation et bienveillance qu'est en fait constituée toute conversation ? D'où, deuxième figure, lorsque le flot de paroles se retourne en silence obstiné. Il s'agit toujours de vaincre, et celui qui l'emporte n'est pas forcément le plus prolixe :

Bakhtiarévitch se taisait et ce silence [...] provoquait Galous et le poussait à élever la voix [...]. Tandis que Bakhtiarévitch se taisait, la voix de Galous commençait à baisser. [...] Et enfin, elle se perdit dans le grand calme de la nuit. Maintenant les deux jeunes gens se taisent. Et cependant dans la nuit pesait le lourd et obstiné silence de Bakhtiarévitch. [...] Par le poids même de son existence, il démentait résolument tous les propos de Galous et exprimait sa pensée muette, claire et immuable (Andric, 1961 : 259).

L'art de la conversation est bien souvent une guerre, où chacun use de ses armes propres, du verbiage le plus échevelé au mutisme le plus buté. Nul n’a mieux rendu compte de ces combats, souvent impitoyables, que Nathalie Sarraute. Soulever la «chape de plomb » du langage pour dévoiler la « sous-conversation», faite de blocs de silence qui s'affrontent, telle fut son entreprise. Martereau décrit ainsi le « dialogue » d'un père et de sa fille. Le premier, «s'installe dans ce silence tel un vieux récidiviste qui retrouve aussitôt, chaque fois qu'on l'y ramène, ses habitudes de prison ". Puis, vases communicants, le silence glisse à la seconde : "Elle fait fonctionner ce que j’appelle son système de pompe : son silence devient plus dense, plus lourd, [...] nos mots sont aspirés par lui, [...] et vont s’écraser quelque part en elle [...] happés par son silence » (Sarraute, $1964: 124)$. Conversation ? Jeu du qui perd gagne : c'est à qui écrasera l'autre du poids de son silence.

Pour terminer sur les formes que prend la conversation, on peut encore évoquer ceux qui n'y prennent pas part, soit qu'ils sont atteints de cette maladie dite de l'esprit de l'escalier :

Les silencieux, les muets, ceux qui ne savent pas raconter, [...] qui ne trouvent à chaque fois la réplique adéquate qu’une fois rentrés chez eux ; [...] leurs visages ne semblent-ils pas plus expressifs, beaucoup moins vides que les autres ? À croire que, sur ces visages, grouillent les lettres de tous les mots des histoires qu'ils n'ont pu raconter (Pamuk, 1994 : 282).

Soit qu'ils n'ont, strictement, rien à dire : ils ne pensent pas, comme le Grand Dauphin sous la plume acerbe de Saint-Simon, tellement paresseux et « avare au-delà de toute bienséance " qu'il est "silencieux jusqu'à l'incroyable, absorbé dans sa graisse et dans ses ténèbres » $(1975: 77)$. 
Toute conversation est ainsi entrelacée de silences, qui la plombent comme chez Sarraute, mais qui l'approfondissent aussi, en la détournant de son cours. Car pour être évitements ces non-dits ne sont pas toujours hostiles - ils peuvent même être, sinon le signe d'une communauté retrouvée, du moins d'un accord tacite. Il m'est ainsi heureux d'imaginer certains dialogues d'écrivains. Carl Seeling rend compte de sa première rencontre avec Walser : "L'aînée des sœurs de Robert, Lisa, m'avait rendu attentif au fait que son frère était excessivement méfiant. Que devais-je faire ? Je fis silence. Il fit silence. Et c'est sur cette étroite passerelle de silence que nous nous rencontrâmes " (1992: 10). Sur une même passerelle se tiennent Joyce et Beckett ( 25 ans d'écart, si proches, si différents - si faits pour s'entendre) : le second, qui débarque à Paris, " aimait le silence comme Joyce; ils avaient des conversations qui consistaient souvent en silences échangés de l'un à l'autre " (Ellmann, $1987: 2,297)$.

\section{Le monde du silence}

Le monde entier est une parole de l'insaisissable adressée à notre âme ou une parole de notre âme adressée à elle-même (Hofmannsthal, 2005 : 126).

Continuons à tendre de telles passerelles entre écrivains, cette fois sur le registre de la relation au monde. Les interrogations sont les mêmes : nous parle-t-il avec bienveillance ou nous est-il inamical ? Ou bien, «bloc de silence qui ne se laisse pas pénétrer, qui ne laisse rien pénétrer " (Michaux, 1976 : 11), est-il le grand Autre ? Balancent ainsi les écrivains, du désenchantement qui se pense lucide au mysticisme le plus extatique, de l'espérance que du monde une parole s'adresse à nous au soupçon qu'il n'y a là que solipsisme. Cette incertitude peut générer une angoisse aux accents métaphysiques :

Quelle parole a surgi près de moi,

Quel cri se fait sur une bouche absente?

$[\ldots]$

Pourtant ce cri sur moi vient de moi,

Je suis muré dans mon extravagance.

Quelle divine ou quelle étrange voix

Eût consenti d'habiter mon silence? (Bonnefoy, 1998 : 79)

D'aucuns n'ont pas ces doutes : « Nature profonde et neutre, que ne hante aucun esprit malin ni aucune bienveillance mystérieuse » (Peña-Ruiz, 2001 : 59). Naîtrait ainsi une nouvelle sérénité : cette absence radicale, "l'homme d'aujourd'hui ne l'éprouve plus comme un manque, ni comme un déchirement. Devant un tel vide, 
il ne ressent désormais nul vertige " (Robbe-Grillet, $1963: 64$ ). La cause semble donc désormais entendue : le monde se tait, non parce qu'il entend garder une part de mystère, mais parce qu'il n'a, strictement, rien à dire : "L'univers est muet, il n'a jamais parlé à personne, même si un nombre incalculable dêtres humains tentent quotidiennement de l'interpeller, par des prières, des interrogations philosophiques " (Amari, $2014:$ 141).

Rien ne répond donc plus à l'attente de l'homme qui interroge la nature, et il faudrait alors s'y résoudre : le temps où les dieux se mêlaient de l'histoire des hommes, leur adressant injonctions et conseils, est révolu. Au monde, ils ne prêtent plus leur voix ; le quittant, ils se sont tus. L'homme, orphelin, s'en est trouvé désemparé :

À partir de quel moment les dieux n'ont-ils plus voulu de nous comme spectateurs, comme participants ? Nous avons été abandonnés à nous-mêmes, à notre solitude, à notre peur, et le problème est né. Qu'est-ce que le monde ? Qui sommes-nous? (Ionesco, 1968 : 167).

La quête devient purement métaphysique? «Le silence déraisonnable du monde » (Camus, 1979 : 44) est aussi pour l'homme ouverture à tous les possibles. Lorsqu'un Théodore Monod, par exemple, évoque " les grands horizons dévastés " du désert, "sans limites, [...] grandioses dans leur tragique et inhumaine immensité », il met certes en évidence notre petitesse, mais sans pour autant que cela se teigne de désarroi - plutôt d'une calme modestie : "Ici, nous sommes[...] ignorés avec une sereine indifférence ; [...] il est bon, parfois, de se l'entendre répéter par quelque coin de nature sauvage, vierge, et qui ne ment pas " (2003 : 125). Cette modestie conduit même certains à se fondre dans le vertigineux mutisme du monde. Le héros du Dit de Tianyi, roman de François Cheng, est relégué dans un camp du Grand Nord chinois. Dans ces espaces infinis, si rudes, « on se laisse prendre par l'immense vide, et l'on se sent un moment la part la plus silencieuse, la plus immobile de l'univers » (1998: 367).

Empreinte de sérénité ou au contraire de déréliction, cette idée d'un silence total du monde n'est pourtant pas la plus répandue. Nombreux sont ceux qui croient entendre la voix silencieuse du monde : " Il se sentit tout à coup plein de silence. Il avait coulé sans bruit dans son esprit comme un robinet ouvert» (Durrell, 1965 : 206). Capter ces voix, comme avec la harpe éolienne antique, vibrante, résonnante de ces sons, est-ce alors possible? Certains le pensent : le monde nous parle, son «silence essaie de captiver le passant» (Kierkegaard, $1979: 17$ ). Mais faire ainsi de «chaque atome de silence [...] la chance d'un fruit mûr » (Valéry, $1957: 55)$ n'est pas donné à n'importe quel passant, "le poème de la création est ininterrompu ; mais rares sont les oreilles qui l'entendent» (Thoreau, 2017 : 97). « Je m’éveillai dans la Nature et la lumière, où toute interrogation trouve sa réponse » $(2017: 311)$ : une telle extase n'est promise qu'aux initiés. 
Le monde ainsi joue avec nous de ses silences, multipliant ses modes d'apparition : " Le silence [...] s'attarde sur les objets les plus insignifiants, [...] il se cache dans notre main qui se referme, dans le pli d'un tissu, dans les tiroirs où rien ne loge sauf des épingles, des boutons » (Lobo Antunes, 2013 : 155). Loin de nêtre qu'absence de bruit, selon la pauvre définition des dictionnaires, comme l'air est traversé de courants sans cesse changeants (de force, de direction), le silence est parcouru de vibrations. Chaque endroit du monde a ainsi son silence propre, au rythme singulier, comme le village de l'enfance de Gabriel García Márquez, dont, écrit-il, « j’aurais pu reconnaître le silence tangible les yeux bandés parmi tous les silences du monde » $(2003: 31)$.

Il semble toutefois que, dans certaines contrées, ces pulsations du silence se fassent plus perceptibles. À commencer, on l'a vu avec Monod, par le désert. Pendant les quarante jours où le Christ s'y retire, «le silence résonne dans ses oreilles avec un bruit de coquillages " (Saramago, $2000: 278$ ) - est-ce ce silence bruissant, rythmé qu'il y venait chercher? Tant d'autres ont tâché de percevoir ce murmure, sourd et pourtant ouvert sur l'immensité, du désert - avec, toujours, ce léger doute qui subsiste : et si tout cela, construction humaine, n’était qu'illusion : "Les déserts sont des créations du silence, qui tendent à éteindre dans le plat des sables et le transport mystique ce qui fut d'abord une aspiration inquiète à s'élever » (Glissant, 2005 : 92). Pire : et si ce n’etait qu'hostilité ? Et si le bruit de coquillages entendu par le Christ de Saramago ne suscitait qu'angoisse : « Le silence bruissant dans les oreilles comme lorsqu'on écoute la mer dans un coquillage [...] me paraissait angoissant et horrible (Sebald, 2016:203) ?

On retrouve le même doute avec d'autres paysages - ainsi la mer : «La mer s’étale, pâle et scintillante, elle est sans voix [...]. Les petits écueils et les récifs qui s'avancent dans la mer [...], tous sont sans voix. Ce monstrueux mutisme qui fond soudain sur nous est beau et terrifiant " (Nietzsche, 1980 : 301). Là aussi pourtant, de ce silence d'autres s'imprègnent, y trouvent comme une paix souveraine : "C'était l'envers du monde. Une nuit lumineuse et calme vous portait, foisonnante des algues calmes et glacées du silence " (Duras, 1987 : 130). La mer, et plus encore l’océan, communiquent ainsi leur infini balancement à ceux qui contemplent, fascinés et effrayés à la fois, la falaise balayée des vagues, les jeux de silence et de fracas naissant, mourant l'un de l'autre, immobilité et mouvement mêlés :

L'Océan brille et jaillit, [...] se fracasse contre la falaise - l'écume s’éparpille [...], une seconde de répit : un fracas, un tonnerre, un tumulte tellement vastes qu'ils se muent en silence. Silence. [...] Immobile, le scintillement de l'infini miroir. Mouvement figé : passion de l'éternité (Gombrowicz, 1995 : 368).

Ce mutisme des choses nous subjuguerait-il tant que nous en finissons par épouser sa cruauté ? L'interrogation demeure, au risque, peut-être de la surhumanité nietzschéenne : «Oh mer ! Oh soir ! [...] Vous apprenez à l'homme à cesser d'être homme ! [...] Doit-il devenir comme vous êtes à présent, pâle, scintillant, muet, monstrueux, reposant en soi-même ? Elevé au-dessus de soi ?» (1980:301-302). 
Autre élément naturel, la neige, avec laquelle il semble qu'enfin on trouve une vraie sérénité : elle " apaise tout, on dirait qu'elle porte en elle le silence ou, plutôt, que dans l'espace qui sépare deux flocons, entre les flocons, il y a le silence " (Stefànsson, $2011:$ 88). Ce silence dont elle enveloppe toute chose a aussi la capacité d'amortir les sentiments trop exacerbés : " Si la neige, à nouveau, pouvait tout envelopper, effacer les reliefs et les chagrins, taire les mots » (Jourde, 20014 : 97). Orhan Pamuk, le grand écrivain turc, consacre un de ses romans à cette qualité singulière d'un paysage enneigé de permettre à la parole poétique, indissociable du silence dont elle émerge, de s'éveiller. À Kars, aux confins de l’Arménie et du Kurdistan, deux silences s'opposent : celui imposé par le pouvoir central d'Ankara, redoublé par celui des intégristes musulmans et des nationalistes kurdes, et celui du manteau de neige qui recouvre tout. C'est ce dernier qui va l'emporter : ce « rideau de tulle [...] qui rappelait le silence d'après la fin du monde » ouvre les vannes de la poésie : "Au début d'un poème, il aurait qualifié ainsi l'état de ses sentiments, de " silence de neige " (2007 : 367, 11). C'est qu'elle éteint tous les bruits parasites, sons et paroles superflues : "Comme sur les lignes et les couleurs et les perspectives, la neige était aussi tombée sur les bruits et même sur la possibilité de faire du bruit : dans cet espace capitonné, les sons ne vibraient plus »(Calvino, $1981: 30-31$ ).

Ouvrant la voie à la parole poétique ? Comme pour le désert et la mer, d’aucuns n’ont pas cette vision de la neige, n'y voyant qu'indifférence dédaigneuse : « Il s'apercevait que la neige menait une vie à elle, exactement comme la terre ou la mer : mais une vie stérile, secrète, couleur d'argent » (Prokosch, $1980: 220$ ).

Mer, désert, neige : éléments paysagers associés au silence... Des temps aussi le sont - et d'abord la nuit. Déjà, aux heures déclinantes de la journée, on dirait que le soleil décide de modifier ses accords, qu'aux bruits diurnes il préfère maintenant les plages de silence du crépuscule, «silence étonnant qui semblait toujours choisir ce moment pour venir se poser sur le fleuve et sur les roseaux, parmi les derniers oiseaux encore éveillés » (Gary, 1967 : 54). Puis vient la nuit, ultime complice du silence. Il se love en elle, lui ouvre des espaces où il peut s'épanouir, accentué même par les quelques sons qui subsistent :

Ce n'est que la nuit que les sons trouvent leurs places, [...] le silence qu'ils portent autour d'eux décrit l'espace, le tableau noir de l'obscurité est marqué par des points et des traits sonores, les aboiements mouchetés d'un chien, la chute estompée d'une vieille feuille de palmier (Calvino, 1991 : 173).

La nuit, tout se ligue pour faire silence, lui permettre d'envahir l'âme des hommes. Il s'insinue partout, engendrant une plénitude exprimée par un écrivain vietnamien : "Une nuit paisible. [...] Le silence coule sur le feuillage argenté des orangers, sur les plantes grimpantes au pied du mur » (Duong, 2006 : 119), comme par un écrivain français : « Un silence infini s'instaure, impérial. De temps en temps, on entend une goutte de rosée tomber sur une feuille de bananier ». C’est Legrandin qui parle, 
écoutant « la musique que joue le clair de lune sur la flûte du silence» (Proust, 1969 : 127). Le même Proust, on le sait, a dit aussi l'angoisse qui vient avec le silence nocturne - ces peurs «du noir » que les âmes simples, enfants, animaux, éprouvent plus particulièrement : «Les renards du désert glapissent comme des démons quand vient la nuit ; sais-tu pourquoi ? Pour rompre le silence qui les terrifie » (Vargas Llosa, $1984: 21)$.

Vient enfin le matin qui, certes, apaise les frayeurs de la nuit, mais étouffe aussi l'aménité de son silence : "Comme un point d'impact en transparence sous la dernière vapeur de la nuit, [...] le petit jour écarquille son œil ensommeillé. [...] En bas, à ras de terre, la nuit se tasse, encore compacte [...]. Le ciel s’épluche. Pureté du jour ». C'en est fini de la paix nocturne : « Les bruits éclatent. Tintamarre. Moteurs. Ferraille. La talonnade de la foule broie la croûte du silence» (Calaferte, $2014: 131$ ).

\section{Conclusion}

Les mots, gouttes de silence à travers le silence (Beckett, 1971 : 159).

Chaque silence, on l'a vu, a deux faces, l'une positive, l'autre négative, et pose, lancinante, la même interrogation : Ne serait-ce pas nous qui inventons la voix du monde ? La question rejoint celle, plus large, de la représentation, cette "structure d'intelligibilité, [...] « médiation imaginaire entre la conscience et le monde dont il est impossible de s'abstraire» (Glaudes, 1999: XXI). Imaginaire ? La médiation vient alors bien de nous. Obstinément silencieux, le monde reste l'Autre absolu - ou, dirait Schopenhauer, il (n') est (que) notre représentation.

Dualisme aux accents vaguement kantiens ? La pensée mystique, fût-elle sans dieu, le réfute. Dans l'extase, l'hors de soi, on est complètement pris dans la sphère du monde : envahi, gagné par le monde, l'extatique épouse son silence. On ne tombe plus dans le silence, c'est lui qui se lève, aurore créatrice d'un nouveau jour, celui de la connaissance :

Le silence [...] est créateur. Il est énergétique. [...] La racine de la connaissance ne peut être que dans le silence. Le papillon connaît la flamme au moment où la flamme le brûle, et à ce moment où il connaît la flamme, brûlé, il ne peut ni penser ni dire. C’est une image de la connaissance. [...] Le papillon ne peut raconter l'expérience de la flamme. Le silence seul (Régy, $2007: 67-68)$.

Suit pourtant le langage, nommant la rencontre des choses : « Rien n'est sans langage. [...] Le mot dit à la chose qu'elle manque et il l'appelle [...]. Comme si ce mouvement amoureux de la parole avait appelé le monde » (Novarina, 2010 : 33). Bien 
sûr, n'importe quel verbe ne saurait convenir à une telle entreprise, simultanément appel aux choses et réponse à leur silence. Pour tourner les difficultés, il faut user de subterfuges : métaphores, images, oxymores - on a évoqué à plusieurs reprises des silences parlants, il y a aussi des «bruits muets " (Corbin, $2016: 111$ ). Comment éviter de telles tournures, dès lors qu'il s'agit de "parler du silence " ? Un halo de silence entoure les mots, menacés de s'éteindre dans le silence qui les suit ou, pire, d’en être eux-mêmes emplis, lieux-communs, clichés qui à leur tour étouffent tout vrai silence... Le balancement incessant entre le monde et le moi - c'est que le monde, nous en faisons partie - ces affinités, comment les donner à voir, sinon en contournant les obstacles que les mots du dictionnaire dressent sur la route des poètes à l'aide des figures de rhétorique, seules capables d'entrouvrir la muette coquille du monde?

Il y a ainsi des points de contact, d'adhérence selon un terme de topologie mathématique, entre langage et silence. La tâche essentielle des écrivains est de s'approcher au plus près de ces points où la parole fait mine de rompre le silence, où le silence, tout aussi bien, rompt la voix, où s'accordent rythmes humain et "mondain ", où " une sève humaine " s'accorde " en profondeur aux saisons, aux rythmes de la planète " (Gracq, $1995: 101)$ : "Je voudrais surtout parler du silence. Un silence qui est [...] un rapport d'égalité actif entre le monde et l'homme. [...] Ce qui compte, c'est cette harmonie des rythmes " (Le Clézio, 1985 : 141).

Cet accord, ce serait ce qu'on appelle l'esthétique :

L'esthétique est une relation qui s'établit entre lêtre humain et une certaine combinaison de formes. Ici, nous pouvons [...] avancer le terme de résonance, dans le sens où il désigne un phénomène [dans lequel] la vibration excitatrice se rapproche d'une fréquence naturelle de ce système. La sensibilité esthétique est bien une aptitude à entrer en résonance, en « harmonie », en synchronie avec sons, odeurs, formes, images, couleurs (Morin, 1979: 117).

Communion de rythmes, aux frontières du silence ? On n'est, à nouveau, pas très loin d'une certaine expérience mystique, dira-t-on. Mais, et c'est peut-être là toute la différence, elle n'est pas muette, comme celle des mystiques, impuissants devant sa force immense. Tel est le pouvoir, infini en même temps qu'infime, des poètes, au plus près du silence : ils sont, au plus haut point, des esthéticiens.

\section{BIBLIOGRAPHIE}

Amari C. 2014. L'Âne mort. Alger. Barzach.

Andric I. 1961. Il est un pont sur la Drina. Paris. Plon.

Beckett S. 1971. L'Innommable. Paris. Minuit.

Bonnefoy Y. 1998. Poèmes. Paris. Poésie Gallimard. 
Calaferte L. Septentrion. Paris. Folio Gallimard.

Calasso R. 1995. Les quarante-neuf degrés. Paris. Gallimard.

Camus A. 1979. Le mythe de Sisyphe. Paris. Gallimard. coll. «Idées ».

Corbin A. 2016. Histoire du silence. Paris. Albin Michel.

Duong T. H. 2006 Terre des oublis. Paris. S. Wespieser.

Duras M. 1987. Les Petits Chevaux de Tarquinia. Paris. Folio Gallimard.

Durrell L. 1965. Cefalû. Paris. Livre de Poche.

Ellmann R. 1987. Joyce. 2 vol. Paris. Tel Gallimard.

García Márquez G. 2003. Vivre pour la raconter. Paris. Grasset.

Gary R. 1981. Au-delà de cette limite votre ticket n'est plus valable. Paris. Folio Gallimard.

Gary R. 1967. Les racines du ciel. Paris. Livre de Poche.

Glaudes P. 1999. Introduction. In Glaudes P. La représentation dans la littérature et les arts. Anthologie, Toulouse, P. U. M. XXI.

Glissant E. 2005. La Cohée du Lamantin. Paris. Gallimard.

Gombrowicz W. 1995. Journal 1953-1995. Paris. Folio Gallimard.

Gracq J. 1995. Préférences. Paris. Corti.

Hofmannsthal H. 2005. Les mots ne sont plus de ce monde. Lettres à un officier de marine. Paris. Rivages Poche.

Ionesco E. 1968. Présent passé, passé présent. Paris. Mercure de France.

Jourde P. 2014. Pays perdu. Paris. Pocket.

Kierkegaard S. 1979. Étapes sur le chemin de la vie. Paris. TEL Gallimard.

Le Clézio J.M.G. 1985. Le Livre des fuites. Paris. Gallimard.

Lobo Antunes A. 2013. Je ne t'ai pas vu hier dans Babylone. Paris. Seuil. coll. « Points».

Michaux H. 1976. Les ravagés. Montpellier. Fata Morgana.

Monod T. 2003. Méharées. Arles. Actes-Sud.

Morin E. 1979. Le paradigme perdu : la nature humaine. Paris. Seuil. coll. « Points».

Nietzsche F. 1980. Aurore. Paris. Gallimard. coll. «Idées ».

Novarina V. 2010. Devant la parole. Paris. POL.

Pamuk O. 2007. Neige. Paris. Gallimard. coll. « Folio».

Peña-Ruiz H. 2001. Le Roman du monde. Paris. Champs Flammarion.

Prokosch F. 1980. Sept Fugitifs. Paris. Gallimard.

Proust M. 1969. Du côté de chez Swann. À la recherche du temps perdu I. Paris. Gallimard. coll. "Bibliothèque de la Pléiade ».

Régy C. 2007. Au-delà des larmes. Paris. Les Solitaires Intempestifs.

Robbe-Grillet A. 1963. Pour un nouveau roman. Paris. Minuit.

Saint-Simon. 1975. Mémoires. Textes choisis par Galleret P. Paris. 10-18.

Saramago J. 2000. L'Évangile selon Jésus-Christ. Paris. Seuil. coll. « Points ».

Sarraute N. 1954. Martereau. Paris. Livre de Poche.

Sartre J.-P. 1981. Qu'est-ce que la littérature ? Paris. Gallimard. coll. « Idées ».

Sebald. W. 2016. Les Anneaux de Saturne. Arles. Actes-Sud.

Seeling C. 1992. Promenades avec Robert Walser. Paris. Rivages Poche.

Stefànsson J. 2011. La Tristesse des anges. Paris. Gallimard.

Tcheng F. 1998. Le Dit de Tianyi. Paris. Albin Michel.

Thoreau H. 2017. Walden. Paris. Le Mot et le Reste. 
Tsvetaieva M. 2008. Vivre dans le feu. Textes choisis par Todorov T. Paris. Livre de Poche. Valéry P. 1957. Charmes in Euvres I. Paris. Gallimard. coll. "Bibliothèque de la Pléiade ». Vargas Llosa M. 1984. La Ville et les chiens. Paris. Folio Gallimard.

\section{Footbridge. Writer's dialogues about silence}

ABSTRACT: The article deals with the question of the relationship between silence and language among writers. It centers around two themes: first, the place of silence in human relations; second, the silence of the world, or, on the contrary, the voices that spring up from it. This article has a lot of writers debate upon these themes, some being rather perceptive to the positive qualities of the silence of the world and/or of their fellow men, others rather gloomy when seeing theim possibility of hearing the world, or of having more genuine relations with others.

Keywords: silence/language, conversation, word of world. 